\title{
Organic solid-state distributed feedback dye laser with a nonmorphological modification grating
}

\author{
Michifumi Nagawa, Musubu Ichikawa, Toshiki Koyama, Hirofusa Shirai, \\ and Yoshio Taniguchia) \\ Department of Functional Polymer Science, Shinshu University, Ueda 386-8567, Japan
}

Akihito Hongo

Optoelectronic System Laboratory, Hitachi Cable, Ltd., 5-1-1 Hitaka-cho, Hitachi 319-1414, Japan

Shinji Tsuji

Central Research Laboratory, Hitachi, Ltd., Kokubunji, Tokyo 185-8601, Japan

Yoshiaki Nakano

Department of Electronic Engineering, The University of Tokyo, 7-3-1 Hongo, Tokyo 113-8656, Japan

(Received 8 June 2000; accepted for publication 30 August 2000)

\begin{abstract}
We report an organic solid-state distributed feedback laser consisting of an organic active layer and a Bragg grating without morphological change. The active layer consists of 4-(dicyanomethylene)-2-methyl-6-(4-dimethylaminostryl)-4H-pyran as a laser dye, tris(8hydroxyquinoline) aluminum as a host, and poly(methyl methacrylate) as a binder. The threshold and linewidth of the laser are $400 \mu \mathrm{J} / \mathrm{cm}^{2}$ and $1.5 \mathrm{~nm}$, respectively. With this laser, the Bragg grating exists out of the active layer and the grating surface is flat, which are very important for device fabrication and electric driving of the laser. (C) 2000 American Institute of Physics.

[S0003-6951(00)03243-5]
\end{abstract}

Recent advances in the materials and structures of organic light-emitting diodes (OLEDs) (Refs. 1-6) have opened the door to a generation of light-emitting organic devices, namely, organic semiconductor lasers (OSLs). Optically pumped solid-state organic lasers are being investigated by many researchers for the realization of OSLs. ${ }^{7-12}$ Many types of laser structures suitable for organic lasers, for example, microdisks and spheres, vertical-cavity surfaceemitting lasers, distributed Bragg reflectors (DBRs), and distributed feedback (DFB) have been demonstrated. The ultimate goal is to develop a "current-driven" OSL. Therefore, studies on light-pumped organic lasers should consider the aptitude for electric driving.

The efficiency and luminosity of OLED devices have been improved in recent years, making them suitable as the emission mechanism of OSLs. When a resonator is implanted in the OLED, there are many constraints on the resonator. For example, because the OLED consists of thin laminated deposition layers (each about 50-80 nm thick), steps are not allowed on the substrate and the layers must be flat. In addition, the resonator should be separated from the lightemission part to facilitate fabrication.

The DFB resonator is characterized by high efficiency, wavelength selectivity, and ease of fabrication. ${ }^{13}$ The surface, however, is not flat. In this letter, we report an organic solid-state laser consisting of an organic active layer and a DFB resonator without morphological modification.

A Bragg grating in a planar slab waveguide acting as a DFB resonator was fabricated using the planar lightwave circuit (PLC) method ${ }^{14}$ as follows. First, the core layer was deposited by rf sputtering on a silica substrate. In this layer,

${ }^{a)}$ Electronic mail: tany@ giptc.shinshu-u.ac.jp germanium was doped to increase the refractive index. The relative refractive index between the core layer and the silica substrate is $0.8 \%$. The thickness of the core layer is $6 \mu \mathrm{m}$. The Bragg grating is a periodic change in the refractive index of the germanium-doped silica core, fabricated by UV irradiation. Before writing the Bragg grating, the planar slab waveguide was treated with hydrogen loading under high atmospheric pressure to enhance the photosensitivity of the core. The Bragg grating was fabricated in the core layer by irradiating a $\mathrm{KrF}$ excimer laser beam emitting light at 248 $\mathrm{nm}$ through a phase mask. The pitch of the phase mask was $400 \mathrm{~nm}$. A laser beam of $9 \times 3 \mathrm{~mm}$ was scanned, and the grating area was $9 \times 10 \mathrm{~mm}$. After the Bragg grating was imprinted, the grating was annealed at $150{ }^{\circ} \mathrm{C}$ for $4 \mathrm{~h}$ to suppress the shift in the Bragg wavelength that occurs over a long period of time.

The active layer of the device consists of 4 -(dicyanomethylene) -2-methyl-6-(4 - dimethiyaminostryl)$4 H$-pyran (DCM) as a laser dye, tris(8-quinolinolato) aluminum (Alq) as a host material, and poly(methylmethacryrate) as a binder polymer (average molecular weight of 996000 ). The concentration of DCM for the Alq was $3 \mathrm{~mol} \%$, and the mixing ratio of the small molecules to the binder was unity. The active layer was prepared on the Bragg grating by using spin coating. The thickness of this layer was, typically, 320 $\mathrm{nm}$. Figure 1 shows the chemical structures and device structure used in this study.

The active layer was optically pumped with a nitrogen gas laser $(\lambda=337 \mathrm{~nm})$ that generated $500 \mathrm{ps}$ pulses at a 10 $\mathrm{Hz}$ repetition rate. The pump beam was focused to $1 \mathrm{~mm}$ in width and $5 \mathrm{~mm}$ in length on the surface of the active layer by using a cylindrical lens $(f=50 \mathrm{~mm})$. The long axis of the rectangular excitation area was normal to the refractive index 


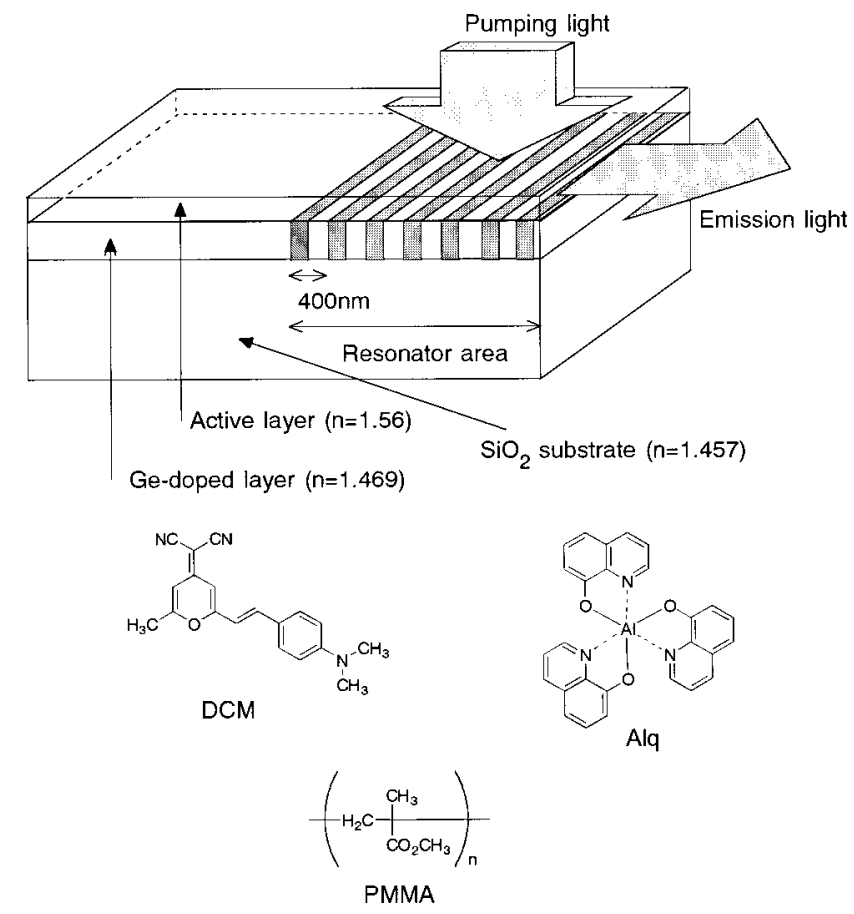

FIG. 1. Schematic illustration of device and structures of chemicals used in this study.

grooves of the grating, and the short side was aligned to a device end. The emitted light was detected at the end face of the device by using a fiber contacting the end face. The detected light was analyzed using a spectrograph (ORIEL MS127i) with an intensified charge-coupled device (ICCD) (Andor ICCD V). The spectral resolution of the measurement system was $0.4 \mathrm{~nm}$.

Emission spectra from the device at excitation intensities of 1300 and $270 \mu \mathrm{J} / \mathrm{cm}^{2}$ are shown in Fig. 2. The spectrum at the lower intensity is a broadband with a peak at $609 \mathrm{~nm}$, and this band is assigned to the spontaneous emission of DCM. The intensity peak wavelength of the band is slightly different from that of the fluorescence peak of DCM in a thin film.

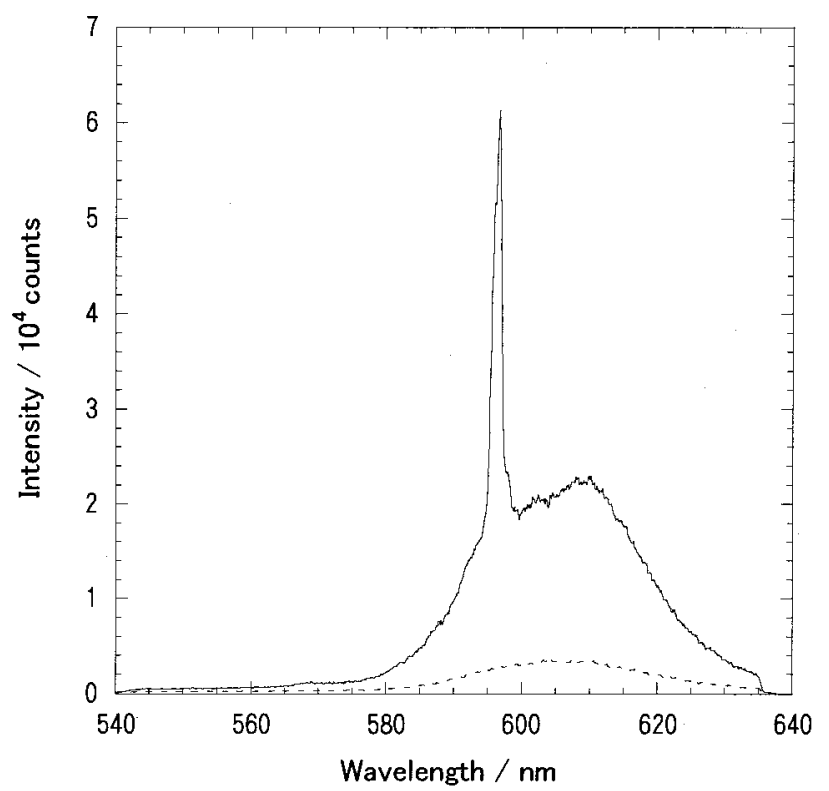

FIG. 2. Emission spectra from the device at excitation intensities of 1400 $\mu \mathrm{J} / \mathrm{cm}^{2}$ (solid line) and $270 \mu \mathrm{J} / \mathrm{cm}^{2}$ (dashed line). $\mathrm{J} / \mathrm{cm}^{2}$ (solid line) and $270 \mu \mathrm{J} / \mathrm{cm}^{2}$ (dashed line). While this bend is not shown in Fig. 3, the threshold is
Downloaded 13 Mar 2003 to 160.252 .124 .1 . Redistribution subject to AlP license or copyright, see http://ojps.aip.org/aplo/aplcr.jsp

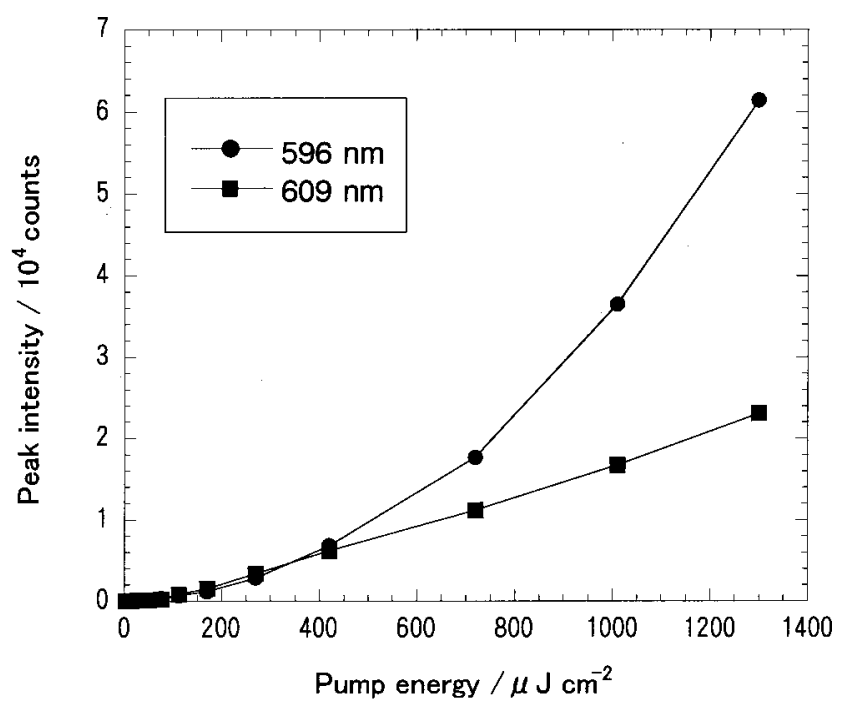

FIG. 3. Excitation intensity dependence of emission intensity at $594 \mathrm{~nm}$ (circles) and $609 \mathrm{~nm}$ (squares).

Emissions were detected at the edge of the planar device, hence, the spectral profile was deformed by the mode distribution of the planar waveguide, which consisted of the substrate, the grating, and the active layer. Therefore, a mismatch occurred. We also experimentally found that the thickness of the device affects the spectral profile deformation and the peak position displacement of the spontaneous emission.

Compared to the spectral shape at the low excitation intensity, that at the high intensity was drastically changed. A very sharp and high-intensity peak came into view at 596 $\mathrm{nm}$ with the background of the spontaneous emission of DCM. The Bragg wavelengths of the resonator $\left(\lambda_{\text {Bragg }}\right)$ can be calculated from the following equation: ${ }^{13}$

$$
\Lambda_{\text {Bragg }}=2 n_{\text {eff }} \Lambda / m
$$

where $n_{\text {eff }}$ is the effective refractive index of the resonator, $\Lambda$ is the period length of the Bragg grating, and $m$ is the order. The $n_{\text {eff }}$ was estimated to be 1.48 by using the transfer matrix method. ${ }^{15}$ Therefore, the resonance wavelength of the device at $m=2$ is estimated to be $592 \mathrm{~nm}$. This value agrees with the wavelength of the line spectrum at the high excitation intensity within the experimental error of the estimated $n_{\text {eff }}$. The linewidth (full width at half maximum) of the sharp peak after subtracting the spontaneous background was only $1.5 \mathrm{~nm}$. These findings strongly suggest that the line at 596 $\mathrm{nm}$ arises from the laser action and that the refractive index grating without morphological change under the active layer acts as a DFB resonator.

The excitation intensity dependencies of the luminescence intensity at 596 and $609 \mathrm{~nm}$ are displayed in Fig. 3. The intensity of the broadband at $609 \mathrm{~nm}$ was proportional to the excitation intensity because of the spontaneous emission. On the other hand, the intensity at $596 \mathrm{~nm}$ radically increased with the excitation intensity. This nonlinear increase based on induced radiation confirms that the line spectrum is determined by the laser action. In general, the intensity dependence bends suddenly at the energy threshold for lasing. 
estimated to about $400 \mu \mathrm{J} / \mathrm{cm}^{2}$ if the threshold is equal to the minimum energy fluence at which the sharp line is observed.

The power threshold of the device was approximately $800 \mathrm{~kW} / \mathrm{cm}^{2}$, a value much larger than that of other recently reported organic solid-state lasers with a grating. For example, McGehee et al. obtained a threshold of $3 \mathrm{~kW} / \mathrm{cm}^{2}$ for a DFB laser using a $\mathrm{SiO}_{2}$ grating and PPV derivatives as the active matter. ${ }^{10}$ Berrgren et al. obtained a threshold of 200 $\mathrm{W} / \mathrm{cm}^{2}$ for a DBR laser consisting of $\mathrm{a} \mathrm{SiO}_{2}$ grating and Alq/DCM2 as the active matter. ${ }^{8}$ These lasers have a morphological grating. Therefore, the relative refractive index between the concave and convex positions of a grating, which is determined from the refractive indices of the $\mathrm{SiO}_{2}$ and active matter, is sufficiently large. On the other hand, the relative refractive index of our PLC grating without morphological changes was only $0.1 \%$. Hence, the diffraction efficiency of our PLC grating is much smaller than that of the other reported gratings. Therefore, the threshold of this laser probably became large.

Two points need to be discussed: (1) the existence of spontaneous background when the device is lasing and (2) the presence of a distinct lasing threshold. When the lasing is sufficient, the induced radiation governs the deactivation processes of the active matter at which spontaneous radiation is suppressed. Thus, spontaneous emission is not ordinarily observed. DFB resonators are not sufficiently built up near the device end because of the disappearance of periodicity. The insufficient feedback region (namely, out of the resonator) also pumped because the end of the optical excitation area was aligned to the device end for experimental reasons. Therefore, spontaneous background was observed when lasing was sufficient and a distinct lasing threshold was not observed.

The final point to discuss is that the device output only a single lasing peak. In general, a double-peaked output with a stop band at the Bragg wavelength appears with simple homogeneous grating DFB lasers, such as that used in this study. ${ }^{10,16}$ However, the device in this study had only a single lasing peak. The reasons for this are not yet clear. One reason may be the low-diffraction efficiency of the PLC grating. Low-diffraction efficiencies induce a narrow-width stop band, and splitting of the two DFB modes with the stop band becomes small. ${ }^{16}$ Therefore, the two DFB modes could not be observed in this study.

In conclusion, we have demonstrated an optically pumped organic solid-state DFB laser consisting of a refractive index grating without morphological fabrication and an organic active layer. This structure is suitable for currentdriven devices. The threshold and linewidth of the laser were $400 \mu \mathrm{J} / \mathrm{cm}^{2}$ and $1.5 \mathrm{~nm}$, respectively. With this laser, the Bragg grating exists out of the active layer and the grating surface is flat, which are very important for device fabrication and electric driving of the laser.

This work was supported by a grant-in-aid for COE Research (10CE2003) from the Japanese Ministry of Education, Science, Sports, and Culture.

${ }^{1}$ C. W. Tang and S. A. VanSlyke, Appl. Phys. Lett. 51, 913 (1987).

${ }^{2}$ C. Adachi, T. Tsutsui, and S. Saito, Appl. Phys. Lett. 55, 1489 (1989).

${ }^{3}$ Y. Kuwabara, H. Ogawa, H. Inaba, N. Noma, and Y. Shirota, Adv. Mater. 6, 677 (1994).

${ }^{4}$ P. E. Burrows and S. R. Forrest, Appl. Phys. Lett. 64, 2285 (1994).

${ }^{5}$ J. Kido, K. Kimura, and K. Nagai, Science 267, 1332 (1995).

${ }^{6}$ A. Yamamori, C. Adachi, T. Koyama, and Y. Taniguchi, Appl. Phys. Lett. 72, 2147 (1998).

${ }^{7}$ V. Bulovic, V. G. Kozlov, V. B. Khalfin, and S. R. Forrest, Science 279, 553 (1998).

${ }^{8}$ M. Berggren, A. Dodabalapur, R. E. Slusher, and Z. Bao, Synth. Met. 91, 65 (1997).

${ }^{9}$ J. A. Rogers, M. Meier, and A. Dodabalapur, Appl. Phys. Lett. 73, 1766 (1998).

${ }^{10}$ M. D. McGehee, M. A. Diaz-Garcia, F. Hide, R. Gupta, E. K. Miller, D. Moses, and A. J. Heeger, Appl. Phys. Lett. 72, 1536 (1998).

${ }^{11}$ M. Nagawa, O. Tsuzaki, Y. Okumura, C. Adachi, T. Koyama, and Y. Taniguchi, in Proceedings of the International Conference on Advanced Fiber Materials, Ueda (1999), p. 310 (unpublished).

${ }^{12}$ Y. Okumura, M. Nagawa, C. Adachi, M. Satsuki, S. Suga, T. Koyama, and Y. Taniguchi, Chem. Lett. 754 (2000).

${ }^{13}$ R. Hofmann, H. P. Gauggel, U. A. Griesinger, H. Grabeldinger, F. Adler, P. Ernst, H. Bolay, V. Harle, H. Schweizer, and M. H. Pikuhn, Appl. Phys. Lett. 69, 2068 (1996).

${ }^{14}$ H. Uetsuka, H. Arai, K. Tamura, H. Okano, R. Suzuki, and S. Kashimura, IEICE Trans. Electron. E81-C, 1205 (1998).

${ }^{15}$ L. M. Walpita, J. Opt. Soc. Am. 2, 595 (1985).

${ }^{16}$ K. Utaka, S. Akiba, K. Sakai, and Y. Matsushima, IEEE J. Quantum Electron. QE-20, 236 (1984). 